\title{
Multidrug-resistant phenotype and isolation of a Novel SHV- beta-Lactamase variant in a clinical isolate of Enterobacter cloacae
}

\author{
${\text { Amel Bourouis }{ }^{1 *}, \text { Mouhamed Ben moussa }^{2} \text { and Omrane Belhadj }}^{1}$
}

\begin{abstract}
Background: ESBL-producing bacteria are a clinical problem in the management of diseases caused by these pathogens. Worldwide, systemic infections with BL enzymes are evolving by mutations from classical bla genes in an intensified manner and they continue to be transferred across species.

Results: E.cloacae BF1417 isolate and its transconjugants gave positive results with the DDST, suggesting the presence of ESBL. Sequence analysis revealed a bla $a_{\text {SHVESBL}}$-type gene that differs from the gene encoding SHV-1 by five point mutations resulting in three amino acid substitutions in the coding region: C123R, I282T and L286P. This novel SHV-type enzyme was designated SHV-128. The conjugation tests and plasmid characterization showed that the bla $a_{\mathrm{SHV}-128}$ is located on a conjugative plasmid IncFIl type. Expression studies demonstrated that the above mutations participated in drug resistance, hydrolysis of extended spectrum $\beta$-lactam and the change of the isoelectric point of the protein.

Conclusion: These findings underscore the diversity by which antibiotic resistance can arise and the evolutionary potential of the clinically important ESBL enzymes. In addition, this study highlights the need for systematic surveillance of ESBL-mediated resistance as well as in clinical areas and communities.
\end{abstract}

Keywords: Bacterial resistance, Enterobacter cloacae, ESBL, SHV-128, Mutations

\section{Background}

In Gram-negative bacteria, ESBL-mediated resistance is emerging worldwide and is mainly due to the mobilization of Ambler class A $\beta$-lactamase, the largest structural/evolutionary group $[1,2]$. Most ESBLs are variants of the classical TEM-1 and SHV-1 ß-lactamases, with one or more amino acid substitutions that confer resistance to broadspectrum cephalosporins and aztreonam. These changes alter the catalytic site allowing the hydrolysis of oxyimino cephalosporins and monobactams $[3,4]$.

SHV $\beta$-lactamases are prevalent in Gram-negative bacteria. These enzymes were originally reported in Klebsiella pneumoniae clinical isolate and they exhibits an overall preference for hydrolysis of sulfhydryl in cephalosporin drug (hence the SHV name) $[5,6]$. SHV-1 can hydrolyse penicillin and cephalosporins but

\footnotetext{
* Correspondence: boursaamel@yahoo.fr

'Address: Laboratory of Biochemistry and Biotechnology, Faculty of Sciences of Tunis, 2092 El Manar II, Tunisia

Full list of author information is available at the end of the article
}

not expanded-spectrum antibiotics such as oxyimino cephalosporins and monobactams. Point mutations in the SHV-1 gene were the first to be reported, and are frequently associated with several mobilization events in bla $_{\mathrm{SHV}}$ gene resulting in new $\beta$-lactamase variants [7]. At present, more than $150 \mathrm{SHV}$ variants have been identified and novel ESBLs continue to be reported at an alarming rate (http://www.lahey.org/studies/). Rapidly, genes encoding these enzymes have been mutated and transferred to other Gram-negative bacteria including Enterobacter cloacae that is commonly found in hospitals and causes a wide range of infections, such as lower respiratory tract infections, urinary tract infections and meningitis [8]. This microorganism is the most commonly isolated member of the Enterobacteriaceae that possess a chromosomally encoded AmpC $\beta$-lactamase that plays an important role in resistance to antibiotics [9]. However, several reports have demonstrated that these species can acquire and express genes encoding extendedspectrum $\beta$-lactamase [10]. 
Until today, $\beta$-lactamase enzymes with an extended spectrum activity against the majority of $\beta$-lactams evolve at an alarming rate and new $\beta$-lactamases that are transferred among species on plasmids with multiple resistance factors are also being described continuously. In this study, we report a phenotypic and molecular characterization of a novel SHV-type $\beta$-lactamase SHV-128 in a multidrugresistant E.cloacae strain.

\section{Methods}

\section{Bacterial strains}

E. cloacae BF1417 was recovered during an epidemiological study at the Military Hospital of Tunis, Tunisia. This strain was isolated from a stool culture of a 75-yearold man hospitalized for renal failure in the intensive care unit at the Military hospital of Tunis. The studied strain was selected on the basis of its multidrug-resistance phenotype (MDR) and it was identified using MALDI-TOF MS system, the VITEK 2 (bioMérieux, La Balme-les-Grottes, France) and the API 20 E system (bioMérieux, Marcy l'Etoile, France). E. coli DH5 $\alpha$ competent cells were used as host for cloning and for the transformation experiments. Streptomycin-resistant E. coli HB101 was used as a recipient for conjugation tests.

\section{Resistance transfer and plasmid characterization}

The transferability of ESBLs genes between the clinical isolate and the recipient was performed by conjugation experiments using the filter-mating procedure [11]. Transconjugants growing on selection plates were subjected to DDST to confirm the resistance transfer and the presence of the ESBL phenotype. Plasmid DNA of the clinical isolate and its transconjugants were extracted with a plasmid extraction kit Promega Midi Plasmid Prep according to the manufacturer's instructions. For determination of plasmid size, the plasmid DNAs from the transconjugants and the clinical isolate were subjected to electrophoresis on $0.7 \%$ agarose gel. Plasmid incompatibility groups were determined by PCRbased replicon typing according to Carattoli et al. [12].

\section{Antimicrobial susceptibility testing and ESBL detection}

The antimicrobial susceptibility of $E$. cloacae BF1417 to $\beta$ lactams, fluoroquinolones, phénicol, aminoglycosides and other drugs was performed on Mueller-Hinton (MH) agar plates by the standard disk diffusion procedure as described previously. Minimum inhibitory antibiotics concentrations were determined by the serial dilution method and results were interpreted according to the Clinical and Laboratory Standards Institute guidelines [13]. ESBL phenotype was confirmed by the double disk synergy test (DDST) in presence of cloxacillin at $250 \mu \mathrm{g} / \mathrm{ml}$ in MuellerHinton agar (Biorad, Marnes-la- Coquette, France).

\section{Analytical isoelectric focusing (IEF)}

The $\beta$-lactamase contents of the clinical strain and its transconjugants were analyzed by isoelectric focusing on a $\mathrm{pH}-3$ to 10 ampholine polyacrylamide (Bio-rad ${ }^{\circ}$, France) gel containing starch $0.5 \%$ at a voltage of 100 to 300 in a 111 Mini IEF Cell (Bio-Rad', France). $\beta$ lactamases with known pIs were used as standards: TEM- 1(pI 5.4),TEM-2 (pI 5.6), TEM-3 (pI 6.3) and SHV- 1(pI 7.6) [14].

\section{$\beta$-lactamase essay and $\mathrm{IC}_{50}$ determination}

E. coli $\mathrm{DH} 10 \mathrm{~B} / \mathrm{PBF} 1417$ was grown overnight at $37^{\circ} \mathrm{C}$ in Trypto-Casein Soy broth (TSB) (Diagnostics, Pasteur, France) supplemented with cefotaxim, $20 \mu \mathrm{g} / \mathrm{ml}$. The cells were harvested by centrifugation and washed once in $25 \mathrm{mM}$ potassium-sodium phosphate buffer ( $\mathrm{pH} \mathrm{7)}$ and resuspended in $1 \mathrm{ml}$ of the same buffer. For preparation of cell free extract, the cells were ruptured by ultrasonic treatment in a UP $400 \mathrm{~S}$ sonicator at $4^{\circ} \mathrm{C}$. Cell debris was removed by centrifugation at 10,000 rpm for $10 \mathrm{~min}$ in a Hettich centrifuge R32 Rotor. The supernatant was loaded onto a Sephadex G-75 column (95 by $2 \mathrm{~cm}$; Pharmacia, Sweden) equilibrated with the same buffer. Eluted fractions were collected and tested spectrophotometrically for $\beta$-lactamase activity with $50 \mu \mathrm{M}$ cephaloridine as the substrate at $255 \mathrm{~nm}$. Active fractions were pooled, dialyzed against $25 \mathrm{mM}$ Tris- $\mathrm{HCl}$ buffer, and then applied to a polyacrylamide slab gel electrophoresis under nondenaturing conditions as previously described [15]. After incubation with cefotaxime $20 \mu \mathrm{g} / \mathrm{ml}, \beta$-lactamase activity was identified as a clear band appeared in the gel background. The detected band was then cut and eluted in $1 \mathrm{ml}$ of the phosphate buffer $(0.25 \mathrm{M} ; \mathrm{pH} 7.4)$ at $4^{\circ} \mathrm{C}$ under moderate agitation overnight. The purity of enzymes was estimated by using sodium dodecyl sulfatepolyacrylamide gel electrophoresis [16]. Hydrolysis of the substrates was monitored by following the absorbance variations as described previously using a CARY 50 Bio UV-visible spectrophotometer connected to a microcomputer. For the partially purified enzyme, relative hydrolysis rate (Vmax) was determined graphically using LinweaverBurk representation [17]. For determination of inhibitor effects, rates of hydrolysis of $1 \mathrm{mM}$ cephalothin were determined in the presence of various concentrations of clavulanic acid and sulbactam. EDTA was used for $1 \mathrm{mM}$ as concentration. In these experiments, the proteic extract was preincubated with the inhibitors for $10 \mathrm{~min}$ before the addition of cephalothin. The inhibitory concentration that allowed the reduction of $\beta$-lactamases activities of $50 \%$ was graphically fixed as previously described [18].

\section{PCR amplification and DNA sequencing}

Detection of gene sequences coding for $\beta$-lactamase enzymes was performed with the plasmid DNA as template 
using primers specific for $b l a_{\mathrm{TEM}}, b l a_{\mathrm{SHV}}, b l a_{\mathrm{CTX}-\mathrm{M}}$, $b l a_{\text {OXA }}$ and AmpC (Table 1). Positive sample was reamplified using Pfu DNA polymerase (Promega, USA). The amplifications were performed on $100 \mu \mathrm{l}$ samples containing $5 \mu \mathrm{l}$ DNA, $0.2 \mathrm{nmol}$ of each primers, $20 \mathrm{nmol}$ deoxynucleoside triphosphate (dNTP), 3 U Pfu DNA polymerase) and $10 \mu \mathrm{l} 10 \mathrm{X}$ buffer (20 $\mathrm{mM} \mathrm{MgSO} 4)$, and dimethyl sulfoxide (7\%). PCR mapping of the genetic environment surrounding the $b l a_{\mathrm{SHV}}$ gene was performed with primers specific for insertion sequence IS26 (primers IS26-1 and IS26-2) combined with forward and reverse primers for $b l a_{\mathrm{SHV}}$ gene as previously described [19]. The experiment was performed on a DNA thermal cycle (AB Applied Biosystem 2720). The PCR products were separated in 1, 2\% agarose gel and visualised with UV. Amplified DNA fragments were sequenced on both strands and the nucleotide sequences and their deduced amino acid sequences were compared with those included in the GenBank database using BLAST software available online (www.ncbi.nlm.nih.gov/BLAST).

\section{Cloning of SHV gene}

The $b l a_{\text {SHV }}$ PCR product was purified using microcolumns of the Microspin Sephacryl S-400 purification system (Amersham Biosciences). Purified DNA were ligated into PGEM-T easy PCR cloning vector and transformed into a competent cell of $E$. coli DH5 $\alpha$ according to the manufacturer's instructions (Promega, WI). Positive colonies were selected on LB agar plates supplemented with ampicillin $(100 \mu \mathrm{g} / \mathrm{ml})^{\circ}$. Plasmids were isolated using the Rapid Plasmid miniprep system and digested with EcoRI restriction enzyme (New England Biolabs) to confirm the presence of the insert. The insert sequences were performed on both strands by using forward and reverse primers: UM11F (5'-CACCTTGCCGACGCAATGAC$\left.3^{\prime}\right)$ and UM11 R (5' -TTAGCGTTGCCAGTGCTCG -3'), an automated fluorescent method based on dye terminator chemistry (AmpliTaq DNA polymerase FS Dye Terminator Cycle Sequencing Ready Reaction Kit; Applied Biosystems) and ABI Prism 3100 automated sequencer (Applied Biosystems, USA). A similarity search of the sequence was carried out using the BLAST program available at the NCBI BLAST homepage (www.ncbi.nlm.nih. gov/blast/).

\section{Nucleotide sequence accession number}

The nucleotide sequence of the $b l a_{\mathrm{SHV}-128}$ gene has been submitted to the EMBL-GenBank database and has been assigned accession number GU932590.

\section{Results}

The clinical strain of E. cloacae BF1417 included in this study was isolated in the ICU of a Military hospital in Tunisia during 2009. Antimicrobial tests showed that the isolate was resistant to all $\beta$-lactams tested except imipenem. Resistance to other drugs, including chloramphenicol, Tetracyclin, aminoglucosides and quinolones, was observed. By filter mating, all the above resistance determinants except cefoxitin, chloramphenicol and Tetracyclin were transferred to E.coli HB101 and were carried on IncFII type plasmid of about 100-kb. The transconjugants expressed high resistance to broadspectrum beta-lactams and gave positive results with the DDST, indicating the production of ESBL. Resistance to other antimicrobial agents was also transferred with the plasmid suggesting their presence in a same genetic context. The MICs of various antibiotics tested for donors, transconjugants, and E.coli HB101 are summarized in Table 2. MICs values revealed similar resistance profiles for most $\beta$-lactams in transconjugants and the clinical strain except the resistance to cefoxitin. When clavulanic acid was combined with cefotaxime and cefdazidime, a reduction in the MICs of these drugs was observed (Table 2). Interestingly, the quinolone resistance phenotype of the transconjugants was identical to this of the donor strain except for nalidixic acid. Indeed, the transconjugants exhibited a reduction in the CMIs from $256 \mu \mathrm{g} / \mathrm{ml}$ to $32 \mu \mathrm{g} / \mathrm{ml}$ which could be due to other factors such as chromosomal gene mutations Such as alterations in the targets of quinolones, and decreased accumulation inside the bacteria due to impermeability

Table 1 Sequences of the primers used to detect $\beta$-lactamases genes

\begin{tabular}{|c|c|c|c|c|}
\hline$\overline{\text { PCR target }}$ & Primer name & Primer sequence $5^{\prime} \rightarrow 3^{\prime}$ & Annealing $\mathrm{T}^{\circ}$ & Ampliquons size \\
\hline \multirow[t]{2}{*}{$\overline{C T X-M}$} & CTX-M-A & CGCTITGCGATGTGCAG & 54 & 539 \\
\hline & CTX-M-B & ACCGCGATATCGTTGGT & & \\
\hline \multirow[t]{2}{*}{ SHV } & SHV-1 F & ATGCGTTATATTCGCCTGTGTAT & 54 & 868 \\
\hline & SHV-1R & TTAGCGTTGCCAGTGCTCGATCAG & & \\
\hline \multirow[t]{2}{*}{ TEM } & TEM-A2 & GTATCCGCTCATGAGACAAT & 54 & 932 \\
\hline & TEM-ext & GTATATATGAGTAAACTTGGTCTG & & \\
\hline \multirow[t]{2}{*}{ OXA } & OXA-1 F & CACAATACATATCAACTTCGC & 54 & 793 \\
\hline & OXA-1R & GTGTGTTTAGAATGGTGATCGC & & \\
\hline
\end{tabular}


Table 2 Minimum inhibitory concentrations (MICs) of various antimicrobial agents obtained for the clinical isolate of E.cloacae and transconjugants

\begin{tabular}{llll}
\hline Antibiotics & \multicolumn{3}{l}{ Minimum inhibitory concentration $(\boldsymbol{\mu g} / \mathbf{m l})$} \\
\cline { 2 - 4 } & E.cloacae BF1417 & E.coli HB101 & HB101x pBF1417 \\
\hline Ampicillin & $>512$ & 4 & $>512$ \\
Ticarcillin & $>256$ & 2 & $>256$ \\
Cefoxitin & $>512$ & 2 & 4 \\
Ceftazidime & 128 & 1 & 128 \\
Ceftazidime + & 4 & ND & 4 \\
ACL & & 1 & 64 \\
Cefotaxime & 64 & ND & 4 \\
Cefotaxime + & 4 & & \\
ACL & & 2 & 8 \\
Cefpirome & 8 & 1 & 256 \\
Aztreonam & 256 & 1 & 32 \\
Nalidixic acid & 256 & 1 & 16 \\
Ciprofloxacin & 32 & 2 & 2 \\
Tetracyclin & $>512$ & 2 & 2 \\
Cloramphenicol & $>256$ & 0,25 & $<2$ \\
Imipinem & $<2$ & & \\
\hline
\end{tabular}

of the membrane and/or an overexpression of efflux pump systems [19].

Analytical isoelectric focusing of crude extract of E.cloacae BxF1417 and its transconjugants revealed the presence of an identical $\beta$-lactamase band with a $\mathrm{pI}$ value greater than 8.6. This activity was revealed with cefotaxime as substrates, which confirms that this was an ESBL.The purity was verified by SDS-PAGE (Figure 1), relative hydrolysis rates of this enzyme for benzylpenicillin, cefotaxime, ceftazidime, and aztreonam are shown in Table 3. The substrate profile of SHV-128 was compared with those of SHV-12 and SHV-2, the first described ESBL. Compared with SHV-12, SHV-128 showed an increasing hydrolysis rate for cefotaxime and, in particular, aztreonam. The hydrolysis rate of ceftazidime was similar to those of SHV-12. However, the Vm value of the SHV-128 enzyme was lower for benzylpenicillin than those of ceftazidime and cefotaxime. Such behavior illustrates well the extended-spectrum activity of SHV-128 enzyme. Inhibition studies showed that SHV-128 was strongly inhibited by clavulanic acid, with IC50 = $4.2 \mu \mathrm{M}$.

Polymerase chain reaction (PCR) amplification did not yield any products except for the bla SHV gene for both, clinical isolate and transconjugants. The PCR products were purified and cloned into pGEM-T Easy cloning vector and transformed into E. coli $\mathrm{DH} 5 \alpha$. The plasmid, pGEM-128, was extracted and the sequence of the PCRgenerated insert was determined on both strands. Sequence data of SHV-128 revealed an ORF of $861 \mathrm{pb}$

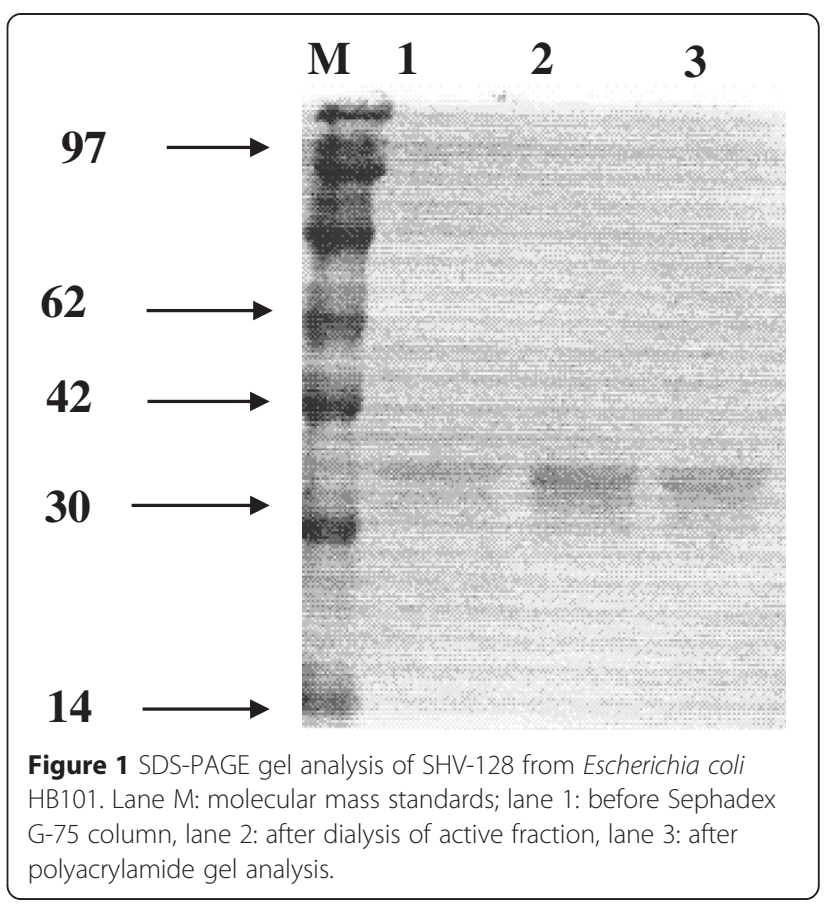

encoding a protein product with 286 amino acid residues. Compared with the reported sequences of bla $a_{\mathrm{SHV}}$ ${ }_{1}$ from GenBank, the resulting sequence revealed a novel SHV-1 variant that was identical to blaSHV-1 except for five point mutations, $\mathrm{A}(351) \mathrm{G}, \mathrm{T}(355) \mathrm{C}, \mathrm{T}(827) \mathrm{C}$, $\mathrm{C}(828) \mathrm{T}$ and $\mathrm{T}(8229) \mathrm{C}$, which was designated SHV-128 (Figure 2). Analysis of the SHV-128 and SHV-1 amino acid sequences revealed 3 amino acid substitutions in the coding region (cystein to arginin at position 123, isoleucine to threonine at position 282 and leucine to proline at position 286). PCR mapping and sequencing revealed that the $b l a_{\mathrm{SHV}-128}$ gene, identified in the clinical isolate ant transconjugants, was enclosed by insertion sequence (IS) IS26 which were detected both upstream and downstream of the reported gene. Thus, the plasmid location of $b l a_{\mathrm{SHV}-128}$ could facilitate its spread especially since this gene is enclosed by insertion sequence (IS) IS26 and associated with other resistant genes.

Table 3 Substrate profiles of SHV-128 compared to those of SHV- 2 and SHV-12 enzymes

\begin{tabular}{llll}
\hline & \multicolumn{2}{l}{ Relative Vmax } & \\
\cline { 2 - 4 } & SHV-2 & SHV-12 & SHV-128 \\
\hline Penzylpenicillin & 100 & 100 & 76 \\
Cephaloridine & 123 & 214 & ND \\
Ceftazidime & $<1$ & 16 & 17 \\
Cefotaxime & 27 & 42 & 56 \\
Aztreonam & 11 & 110 & 140 \\
\hline
\end{tabular}




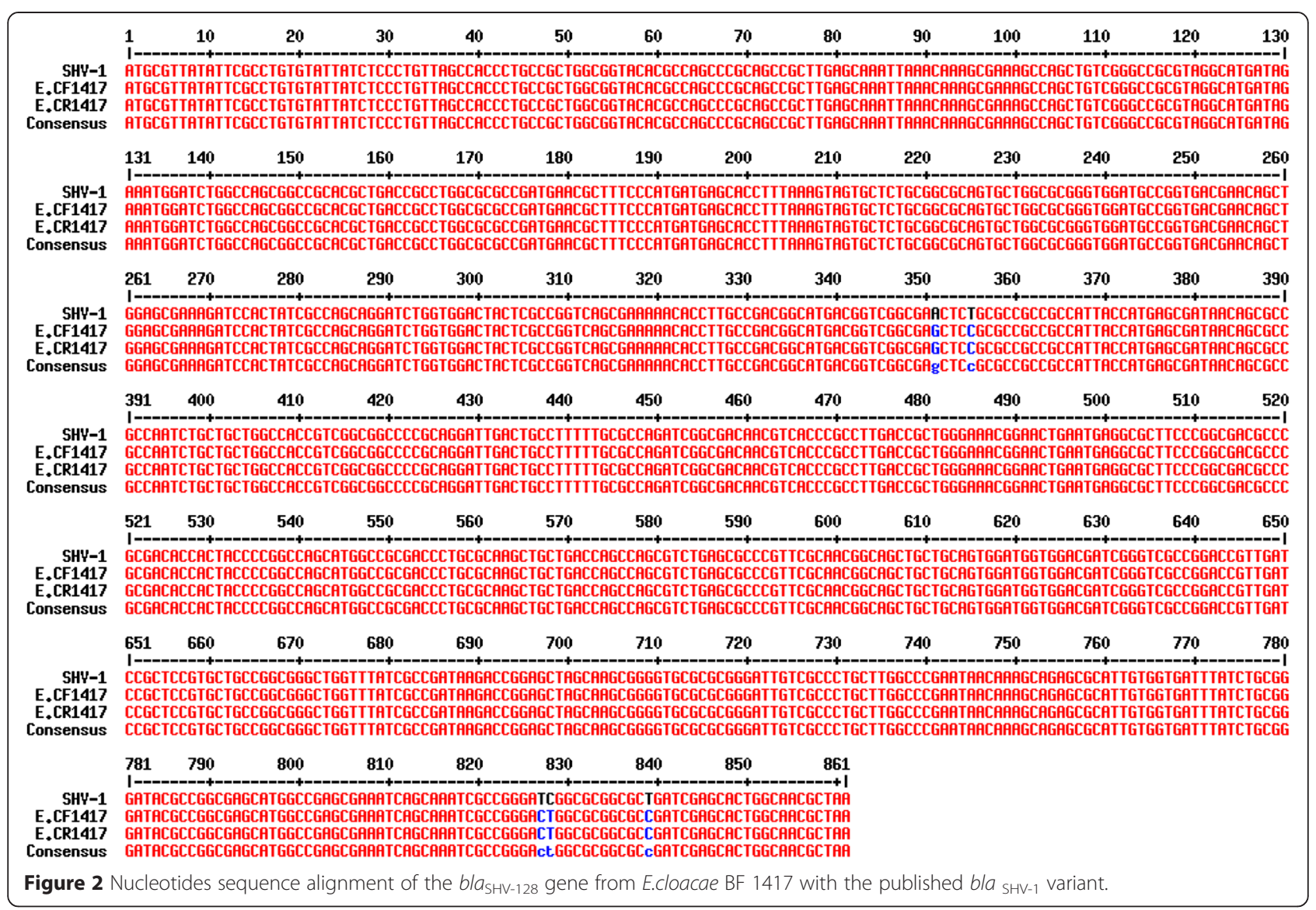

\section{Discussion}

The present study report the phenotypic and molecular characterization of a novel extended-spectrum SHV-type $\beta$-lactamase, designated here as SHV-128. The amino acid sequence of SHV-128 differs from the amino acid sequence of SHV-1 by three amino acid substitutions: cystein for arginin at position 123, isoleucine for threonine at position 282 and leucine for proline at position 286. To our knowledge, these substitutions are observed for the first time in a natural mutant SHV-type $\beta$ lactamase (http://www.lahey.org/studies/). According to the phenotypic analysis, the resulting new enzyme induced a resistance phenotype compatible with that of an ESBL. Indeed, the susceptibility pattern showed that the enzyme caused resistance to C3G such as ceftazidime and cefotaxime and is inhibited by the $\beta$-lactamase inhibitor clavulanic acid. Furthermore, the clinical isolate and the transconjugants gave positive results with the DDST, indicating the production of ESBL. SHV-128 was located on a conjugative plasmid IncFII type of about $100-\mathrm{kb}$. The presence of resistance genes on plasmids and transposable elements allows the genes to be transferred to distantly related bacteria by conjugation, transduction, or transformation [20-23]. In fact, several studies have been conducted to evaluate the effect of promoter mutation or replacement on the ESBL expression, especially those delivered by insertion sequences IS which the case here [24].

Generally, substitutions at positions 240 and 238 seem to be especially critical for ESBL activity and occur in the vast majority of SHV-type ESBL. Indeed, previous experiments on SHV $\beta$-lactamases have reported that G238S and E240K mutations are involved in conferring resistance to C3G [25-27]. On the basis of its amino acid sequence, SHV-128 includes these residus and contains the ${ }^{70} \mathrm{SXXK}^{73}$ tetrad, characteristic of $\beta$-lactamases possessing a serine active site. Two structural motifs characteristic of class A $\beta$-lactamases, were also found to be present in this novel variant: SDN at position 130-132 and KTG at position 234-236 [28]. Also, analysis of SHV-128 structure model (results not cited) showed that C123R mutation is not far from the SDN element that span over active site residues. According to previous studies [29], the arginine residu can induce changes in the electrostatic interactions in an additional manner, which might be a factor in enhancing the stability of the protein. The change of the $\mathrm{pI}$ and the extended spectrum of the enzyme could be attributed to the three natural mutations reported here. However, in order to analyze the role of these substitutions, a site-directed mutagenesis analysis should be done. Furthermore, 
expression and purification of each mutant will be required for better characterization of the SHV-128 enzyme and to specify the effect of each mutation on the active site of the enzyme and its substrate profile. The results obtained in the present study suggest that continuous mutation has led to the occurrence of novel bla $a_{\mathrm{SHV}}$ ESBL gene variants. On the other hand, the presence of the insertion sequences IS suggests that resistance associated with SHV production can contribute to the dissemination of these emerging genes into other nosocomial pathogens.

\section{Conclusion}

In conclusion, the present study explored the molecular basis of antibiotic resistance in a clinical isolate originating from Tunisian hospitals. The data revealed a novel SHVtype $\beta$-lactamase, SHV-128. This protein possessed the conserved class A $\beta$-lactamase motifs and hydrolyzed oxyimino-cephalosporins as well as aztreonam, conferring resistance to these agents. $b l a_{\mathrm{SHV}-128}$ was bounded by IS26, which facilitated its acquisition and its transfer among species in the hospital. These finding highlights the genetic plasticity of the SVH-type. $\beta$-lactamases and the remarkable adaptability of Enterobacteriaceae species to selective antibiotic pressure.

\section{Competing interests}

The authors declare that they have no competing interests.

\section{Authors' contributions}

$A B$ has made substantial contributions to the analysis and interpretation of data, and drafting the manuscript. BM has participated in the delivery of clinical isolates and clinical data. BO has conceptualization of idea and has provided directions for the work. All authors read and approved the final manuscript.

\section{Acknowledgements}

We would like to thank the Ministry of Scientific Research Technology and Competence Development of Tunisia for providing financial support for conducting this research.

\section{Author details}

${ }^{1}$ Address: Laboratory of Biochemistry and Biotechnology, Faculty of Sciences of Tunis, 2092 El Manar II, Tunisia. ${ }^{2}$ Addresses: Laboratory of Microbiology, Military Hospital of Tunis, 1089 Monfleury, Tunisia.

Received: 6 October 2014 Accepted: 25 March 2015

Published online: 11 April 2015

\section{References}

1. El Salabi A, Walsh T, Chouchani C. Extended spectrum $\beta$-lactamases, carbapenemases and mobile genetic elements responsible for antibiotics resistance in Gram-negative bacteria. Critical Reviews Microbiol. 2012:12:1-10.

2. Rayamajhi N, Joo JC, Cha SB, Pokherl S, Shin MK, Yoo YJ, et al. Enzymatic analysis of the effect of naturally occurring Leu138Pro mutation identified in SHV $\beta$-lactamase on hydrolysis of penicillin and ampicillin. BMC Microbiol. 2011:11:1-7.

3. Ben Achour N, Belhadj O, Galleni M, Ben Moussa M, Mercuri PS. Study of a Natural Mutant SHV-Type $\beta$-Lactamase, SHV-104, from Klebsiella pneumonia. Int J Microbiol. 2014;10:1-6.

4. Ramdani-Bouguessa N, Manageiro V, Jones-Dias D, Ferreira E, Tazir M, Caniça M. Role of SHV $\beta$-lactamase variants in resistance of clinical Klebsiella pneumoniae strains to $\beta$-lactams in an Algerian hospital. J Med Microbiol. 2011;60:983-7.

5. Li JB, Cheng J, Wang Q, Chen Y, Ye Y, Zhang XJ. A novel SHV-type $\beta$-lactamase variant (SHV-89) in clinical isolates in China. Mol Biol Rep. 2009;36:1141-8.

6. Gniadkowski M. Evolution of extended-spectrum $\beta$-lactamases by mutation. Clin Microbiol Infect. 2008;14:11-32.

7. Al-Marzooq F, Mohd Yusof MY, Tay ST. Identification of a Novel SHV-B-lactamase Variant (SHV-144) in a Malaysian Multidrug-Resistant Klebsiella pneumoniae Isolate. Jpn J Infect Dis. 2013;66:555-7.

8. Liu W-Y, Wong C-F, Chung KM-K, Jiang J-W, Leung FC-C. Comparative Genome Analysis of Enterobacter cloacae. Plos One. 2013;8:1-15.

9. Liu CP, Wang NY, Lee CM, Weng LC, Tseng HK, Liu CW, et al. Nosocomial and community-acquired Enterobacter cloacae bloodstream infection: risk factors for and prevalence of SHV-12 in multiresistant isolates in a medical centreq. J Hosp Infect. 2004;58:63-77.

10. Szabo D, Melan AM, Hujer MA, Bonomo AR, Hujer MK, Bethel RC, et al. Molecular Analysis of the Simultaneous Production of Two SHV-Type Extended-Spectrum Beta-Lactamases in a Clinical Isolate of Enterobacter cloacae by Using Single-Nucleotide Polymorphism Genotyping. Antimicrob Agents Chemother. 2005:49:4716-20.

11. Bourouis A, Chihi H, Mahrouki S, Ayari K, Ben Moussa M, Belhadj O. Molecular characterization of a transferable blaCTX-M-28 gene in clinical isolates of Enterobacter cloacae. J Microbiol Antimicrob. 2013;5:38-43.

12. Carattoli A. Resistance Plasmid Families in Enterobacteriaceae. Antimicrob Agents Chemother. 2009;53:2227-38.

13. Clinical and Laboratory Standards Institute. Performance standards for antimicrobial susceptibility testing; 20th informational supplement. M100-S20. Wayne, PA: CLSl; 2007.

14. Mahrouki S, Bourouis A, Chihi H, Ouertani R, Ferjani M, Moussa MB, et al. First characterisation of plasmid-mediated quinolone resistance-qnrS1 co-expressed bla $a_{\mathrm{CTX}-\mathrm{M}-15}$ and blaDHA-1 genes in clinical strain of Morganella morganii recovered from a Tunisian Intensive Care Unit. Indian J Med Microbi. 2011;30:437-40.

15. Fereshteh $\mathrm{E}$, Rafiee $\mathrm{R}$. An overlay gel method for identification and isolation of bacterial $\beta$-lactamases. J Microbiol Meto. 2006;64:132-4.

16. Chouchani C, Berlemont R, Masmoudi A, Moreno Galleni M, Frere J-M, Belhadj O. A Novel Extended-Spectrum TEM-Type $\beta$-Lactamase, TEM-138, from Salmonella enterica Serovar Infantis. Antimicrob Agents Chemother 2006;50:3183-5.

17. Ross GW. O'callagham CH. In: Hash H, editor. $\beta$-lactamases asseys Methods in enzymology, vol. XIIII. New York: Academic Press; 1975. p. 69-85.

18. Réjiba S, Limam F, Belhadj C, Belhadj O, Ben-Mahrez K. Biochemical characterization of a novel extended-spectrum- $\beta$-lactamase from Pseudomonas aeroginosa. Microb Drug Resist. 2002;8:9-13.

19. Kim J, Shin HS, Seol SY, Cho DT. Relationship between bla $\mathrm{SHV}_{-12}$ and bla $\mathrm{SHV}_{2 \mathrm{a}}$ in Korea. J Antimicrob Chemother. 2002;49:261-7.

20. Peduzzi J, Barthlmy M, Tiwari K, Mattioni D, Labia R. Structural features related to hydrolytic activity against ceftazidime of plasmid-mediated SHVtype CAZ-5 $\beta$-lactamase. Antimicrob Agents Chemother. 1989:33:2160-3.

21. Matsumoto Y, Inoue M. Characterization of SFO-1, a plasmid-mediated inducible class A $\beta$-lactamase from Enterobacter cloacae. Antimicrob Agents Chemother. 1999:43:307-13.

22. Silva J, Aguilar C, Ayala G, Estrada MA, Garza-Ramos U, Lara-Lemus R, et al. TLA-1: a new plasmid mediated extended-spectrum $\beta$-lactamase from Escherichia coli. Antimicrob Agents Chemother. 2000;44:997-1003.

23. Huang XZ, Frye JG, Chahine MA, Glenn LM, Ake JA, Su W, et al. Characteristics of plasmids in multi-drug-resistant Enterobacteriaceae isolated during prospective surveillance of a newly opened hospital in Iraq. PLoS One. 2012;7:403-6.

24. Gniadkowski M. Evolution of extended-spectrum b-lactamases by mutation. Clin Microbiol Infec. 2008;14:11-32.

25. Matagne A, Lamotte-Brasseur J, Frère JM. Catalytic properties of class A B-lactamases: efficiency and diversity. Biochem J. 1998;330:581-98.

26. Randegger CC, Keller A, Irala M, Wada A, Chler H. Contribution of Natural Amino Acid Substitutions in SHV Extended-Spectrum $\beta$-Lactamases to Resistance against Various $\beta$-Lactams. Antimicrob Agents Chemother. 2000;44:2759-63.

27. Hammond DS, Schooneveldt JM, Nimmo GR, Huygens F, Giffard PM. blaSHV Genes in Klebsiella pneumoniae: Different Allele Distributions Are 
Associated with Different Promoters within Individual Isolates. Antimicrob Agents Chemother. 2005;49:256-63.

28. Singh R, Saxena A, Singh H. Identification of group specific motifs in Beta-lactamase family of proteins. J Biomed Sci. 2009;16:1-7.

29. Deeptak Verma D, Jacobs D-J, Livesay D-R. Variations within Class-A b-Lactamase Physiochemical Properties Reflect Evolutionary and Environmental Patterns, but not Antibiotic Specificity. Plos Comput Boil. 2013;9:1-16.

Submit your next manuscript to BioMed Central and take full advantage of:

- Convenient online submission

- Thorough peer review

- No space constraints or color figure charges

- Immediate publication on acceptance

- Inclusion in PubMed, CAS, Scopus and Google Scholar

- Research which is freely available for redistribution 